\title{
Short-term memory of temporal information revisited
}

\author{
Daniel Bratzke ${ }^{1,2} \cdot$ Rolf Ulrich ${ }^{2}$
}

Received: 22 January 2020 / Accepted: 10 April 2020 / Published online: 28 April 2020

(c) The Author(s) 2020

\begin{abstract}
Previous studies provided diverging evidence regarding modality specificity of temporal information in short-term memory. Some authors reported modality-specific interference effects on visual and auditory duration discrimination, whereas others observed crossmodal interference effects. One reason for these diverging results could be different trade-offs between the temporal discrimination task and the interference task in these studies. Therefore, this study re-examined these effects with interference tasks (speeded color/pitch change discrimination) that were especially suited to assess potential trade-offs between the primary and the secondary tasks. The results showed that the auditory interference task selectively impaired discrimination performance for auditory durations, whereas the visual interference task proved to be inefficient as interference task. The present results agree best with an account that suggests a modality-specific representation of temporal information in short-term memory.
\end{abstract}

\section{Introduction}

How is temporal information encoded and maintained in short-term memory? Contemporary timing theories imply three different hypotheses regarding this question. First, internal clock models (Creelman, 1962; Gibbon, Church, \& Meck, 1984; Treisman, 1963) suggest an abstract and amodal representation (Rammsayer \& Ulrich, 2005; Wearden, Todd, $\&$ Jones, 2006), that is, the number of signals elicited by an internal pacemaker during a certain time interval represent the duration of this interval. This amodal hypothesis appears especially plausible because humans (and animals) can easily compare durations not only within sensory modalities but also across modalities. Second, temporal information might be primarily encoded in the auditory system (crossmodal encoding; Bratzke, Seifried, \& Ulrich, 2012; Guttman, Gilroy, \& Blake, 2005; Kanai, Lloyd, Bueti, \& Walsh, 2011), because the auditory system is especially suited for temporal processing (Welch \& Warren, 1980). Third, intrinsic timing models imply that the short-term representation of temporal information is modality-specific because temporal

Daniel Bratzke

bratzke@uni-bremen.de

1 University of Bremen, Hochschulring 18, 28359 Bremen, Germany

2 University of Tübingen, Tübingen, Germany processing is an inherent feature of early sensory processing (Buonomano \& Karmarkar, 2002; Ivry \& Schlerf, 2008).

An important source of evidence regarding these hypotheses comes from studies investigating modality-specific interference effects on short-term memory of temporal information. In one of these studies, Rattat and Picard (2012) presented unimodal (visual vs. auditory) and bimodal (visual-auditory) durations. To load the visual and the auditory short-term memory sub-systems (i.e., the visuospatial sketchpad and the phonological loop; e.g., Baddeley \& Hitch, 1974) during an 8-s retention interval, they used a visuo-spatial tracking task and articulatory suppression, respectively. In a control condition, participants simply waited for the retention interval to elapse. After the retention interval, participants' memory for the duration was probed by a comparison stimulus that could be shorter or longer than the initial stimulus. The results showed that visual tracking selectively impaired short-term memory of visual durations, and articulatory suppression selectively impaired short-term memory of auditory durations. The authors interpreted this selective interference pattern as evidence for a modality-specific representation of duration information.

Bratzke, Quinn, Bausenhart, and Ulrich (2016) partially replicated the study by Rattat and Picard (2012), focusing on the unimodal conditions and employing a within- instead of a between-subjects design. In contrast to Rattat and Picard, Bratzke et al. observed that articulatory suppression impaired short-term memory not only for auditory but also 


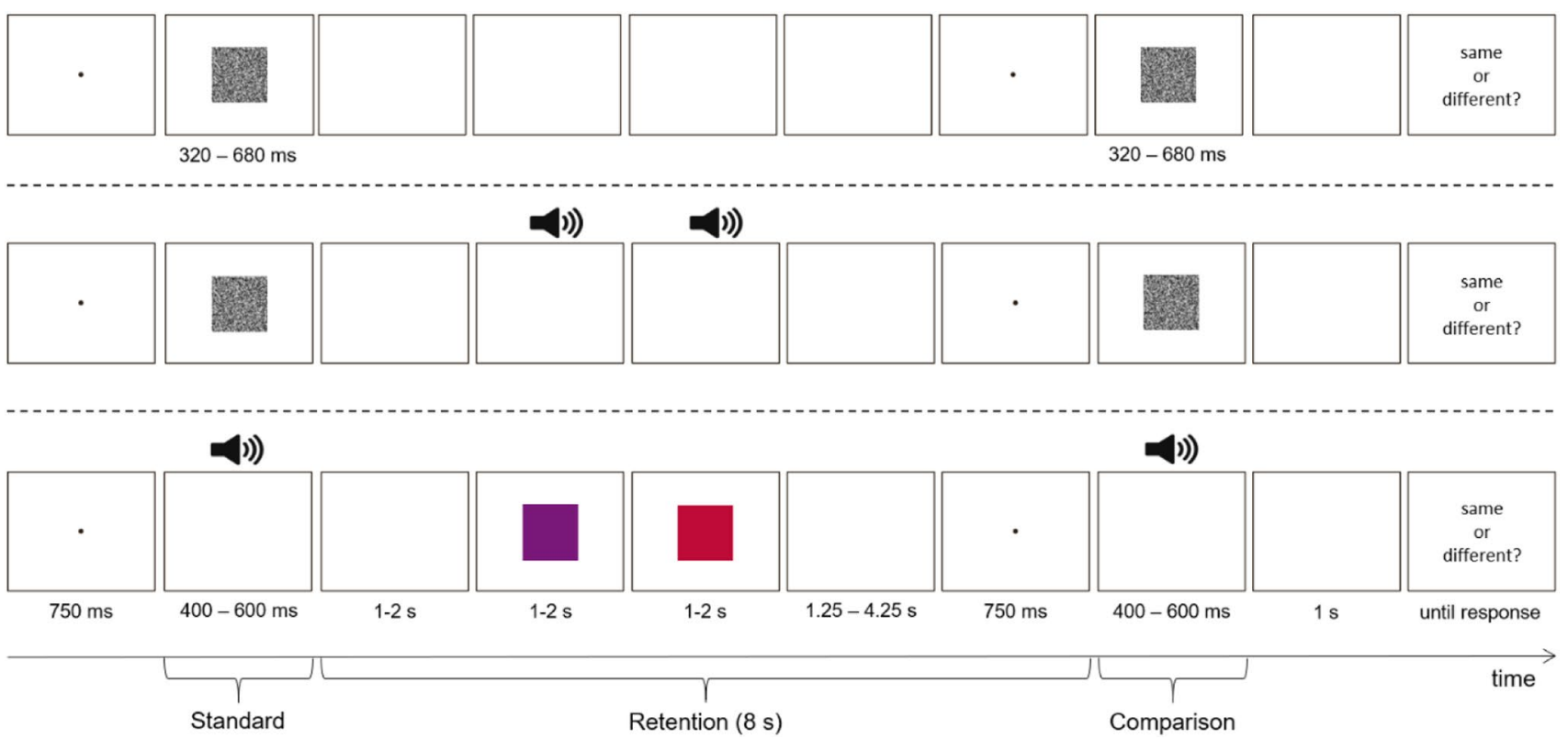

Fig. 1 Time course of three exemplary experimental trials. Upper panel: visual duration discrimination with no interference. Middle panel: visual duration discrimination with auditory interference (pitch discrimination). Lower panel: auditory duration discrimination with visual interference (color discrimination)

performance. Since measuring and analyzing performance in the visual tracking and the articulatory suppression task is rather costly (i.e., it requires eye tracking and video monitoring), we used interference tasks that allow a more efficient way of measurement. The visual interference task (color discrimination) was adopted from Klauer and Zhao (2004). In their study, this task proved to be effective in interfering with short-term retention of unfamiliar Chinese ideographs. The auditory interference task was designed to resemble the visual interference task (pitch discrimination). The time-course of a single trial followed the two previous studies (Bratzke et al. 2016; Rattat \& Picard, 2012), that is, participants had to discriminate two duration stimuli, a standard and a comparison, separated by an 8-s retention interval, which was either empty or filled with an interference task (see Fig. 1). As in the two previous studies, visual and auditory duration discrimination were tested in different sessions, and within each session, the interference tasks were tested in different blocks. As in Bratzke et al. we employed a within-subjects design, that is, all participants performed all conditions.

\section{Method}

\section{Participants}

The final sample consisted of 48 participants (36 women) with a mean age of $23.5(\mathrm{SD}=3.6)$ years. Each participant secondary non-temporal interference tasks and control for possible trade-offs between primary and secondary task 
took part in two separate sessions of the experiment (each lasting about $45 \mathrm{~min}$ ). They received either course credit or payment for their participation. Eight original participants were replaced by new participants because they did not adhere to the instructions or achieved an accuracy in the interference tasks at or below chance level ( $\leq 53 \%$ correct).

\section{Apparatus and stimuli}

The experiment was programmed in MATLAB ${ }^{\circledR}$ using the Psychophysics Toolbox extension (Brainard, 1997; Kleiner et al., 2007). Participants sat in front of a computer screen with a viewing distance of approximately $50 \mathrm{~cm}$. Auditory stimuli were presented binaurally via headphones (Sennheiser HD 380 pro) with a peak amplitude of $60 \mathrm{~dB}$ (A) SPL. White noise (400, 440, 480, 520, 560, and $600 \mathrm{~ms})$ with rise and fall times of $5 \mathrm{~ms}$ served as the auditory duration stimulus in the duration discrimination task. In the pitch discrimination task, there was a reference sine tone of $800 \mathrm{~Hz}$ and 24 test sine tones ranging from 320 to $1280 \mathrm{~Hz}$ (in steps of $40 \mathrm{~Hz}$ with the exception of $800 \mathrm{~Hz}$ ).

Visual stimuli were presented on a 21-inch CRT monitor (Samsung SyncMaster $1100 \mathrm{MB}, 1024 \times 768$ pixels, $150 \mathrm{~Hz}$ ). To make the visual duration stimuli as similar as possible to the auditory ones, we used a square $\left(11.4 \times 11.4^{\circ}\right.$ of visual angle) filled with dynamic grey visual noise (random grey pattern of $256 \times 256$ pixels with $150 \mathrm{~Hz}$ refresh rate) as the visual duration stimulus. As in Bratzke et al. (2016), we used a wider range of durations for the visual $(320,380,440,560,620$, and $680 \mathrm{~ms})$ than for the auditory stimuli (400-600 ms) to compensate for the typically observed poorer temporal sensitivity for visual than auditory stimuli (e.g., Bratzke \& Ulrich, 2019b; Grondin, 1993; Ulrich et al., 2006). A violet-colored (RGB values: 122, 24, 122) square of the same size $\left(11.4 \times 11.4^{\circ}\right.$ of visual angle $)$ served as reference stimulus in the color discrimination task. There were 24 test colors, of which 12 can be categorized as more reddish or bluish than the reference color. The RGB values of the reddish stimuli ranged from $(190,11,55)$ to $(135,22,110)$ and those of the bluish stimuli ranged from $(110,22,135)$ to $(55,11,190)$, with steps of five units for the $R$ and $B$ values and 1 unit for the $G$ value. All visual stimuli were presented at the center of the screen on a white background. Responses were collected using the ' $X$ ' (left index finger) and ' $\mathrm{M}$ ' (right index finger) keys on a standard German keyboard.

\section{Procedure}

Each participant took part in two experimental sessions, a visual and an auditory discrimination session (with a maximum separation of one week between the two sessions). Each trial started with the presentation of a small fixation point at the center of the screen. After $750 \mathrm{~ms}$, the standard duration was presented, chosen randomly with equal probability from the six possible durations. Then, an 8-s retention interval started with a blank screen. $750 \mathrm{~ms}$ before the end of the retention interval the fixation point reappeared and remained at the center of the screen until the presentation of the comparison duration. After the retention interval, the comparison duration was presented. This stimulus was either equal to the standard duration (50\% "same" trials) or it differed (50\% "different" trials). In "different" trials, when the standard duration was shorter than $500 \mathrm{~ms}$, the comparison duration was 120 (240) ms longer in auditory (visual) trials. When the standard duration was longer than $500 \mathrm{~ms}$, the comparison duration was 120 (240) ms shorter in auditory (visual) trials. $1000 \mathrm{~ms}$ after the end of the comparison duration, a prompt message was presented asking the participant to indicate whether the durations of the two stimuli were equal or different. The message included a reminder of the response-to-key key assignment in the duration discrimination task. The next trial started $1000 \mathrm{~ms}$ after the participant's response. For the duration discrimination task, participants were instructed to respond as accurately as possible without any speed restrictions.

Within each modality session (visual vs. auditory duration discrimination), participants completed three blocks of trials, each differing in the nature of the interference task presented during the retention interval. In "no interference" trials, the screen remained empty during the first $7.25 \mathrm{~s}$ of the retention interval. In color discrimination trials (visual interference), the reference-colored square appeared after a random interval between 1 and $2 \mathrm{~s}$ from the start of the retention interval. After another random interval between 1 and $2 \mathrm{~s}$, the square changed its color to one of the 24 test colors. Participants had to indicate whether the color changed toward more reddish or bluish. Irrespective of the participant's response time, the test color stimulus remained on the screen for another random interval between 1 and $2 \mathrm{~s}$. Pitch discrimination trials (auditory interference) followed the same time course as visual ones. In these trials, first the reference tone was played, which then changed to one of the 24 possible test tones. Participants had to indicate whether the tone's pitch changed toward higher or lower. For the interference tasks, participants were instructed to respond as quickly and as accurately as possible to the change of the reference stimulus.

For each of the combinations of duration modality (visual vs. auditory) and interference task (no interference, color discrimination, and pitch discrimination), participants completed three practice trials (randomly chosen from all possible trials within each factorial combination) and four experimental blocks of 12 trials each (one "same" and one "different" trial for each of the possible 6 durations). Within each experimental block, all durations were 
presented equally often and the order of trials was randomized. After each experimental block, participants received feedback about the percentage of correct responses in the duration discrimination task in the preceding block. Duration modality varied between sessions, and interference task varied between blocks within each session. The order of modality sessions and interference tasks was balanced across participants. The response-to-key assignment in the duration discrimination task was also balanced across participants. The response-to-key assignment in the interference tasks was the same for all participants (color discrimination: more bluish-' $\mathrm{X}$ '/more reddish-' $\mathrm{M}$ '; pitch discrimination: lower-'X'/higher-'M').

\section{Results}

As in Bratzke et al. (2016), trials in which RT for the duration discrimination task exceeded $5 \mathrm{~s}$ were regarded as lapses and discarded from all analyses (1.0\% of all trials). As in previous studies (Bratzke et al., 2016; Rattat \& Picard, 2012), for the duration discrimination task the dependent variables were the nonparametric indices $A^{\prime}$ and $B^{\prime \prime}$ for sensitivity and bias (Aaronson \& Watts, 1987; Grier, 1971), respectively. Trials in which participants gave no response in the interference task before the end of the retention interval $(0.4 \%$ of remaining trials) were excluded from these analyses. For the interference tasks, mean RTs (of correct interference trials) and error rates were calculated. Separate ANOVAs with the within-subjects factors duration modality (visual vs. auditory) and interference task (no interference, color discrimination, and pitch discrimination) were conducted for $A^{\prime}, B^{\prime \prime}$ in the duration discrimination task, and for mean RT and error rate in the two interference tasks. The Greenhouse-Geisser correction was used to adjust $p$ values where appropriate.

\section{Primary task performance (duration discrimination)}

Figure 2 depicts $A^{\prime}$ (upper panel) and $B^{\prime \prime}$ (lower panel) values as a function of duration modality and interference task. Note that $A^{\prime}$ can range from 0 to 1 , with $A^{\prime}=0.5$ indicating chance level, and $A^{\prime}=1$ representing perfect discrimination performance. ANOVA revealed a significant main effect of interference task, $F(2,94)=5.56, p=0.005, \eta_{p}^{2}=0.11$. Post hoc Tukey contrasts indicated a reliable difference between pitch discrimination and no interference $(0.71$ vs. 0.76$)$, $p=0.006$, a marginally significant difference between pitch and color discrimination ( 0.71 vs. 0.74 ), $p=0.077$, and no significant difference between color discrimination and no interference ( 0.74 vs. 0.76$), p=0.639$. This pattern suggests that only pitch discrimination interfered with shortterm memory of temporal information. The main effect of
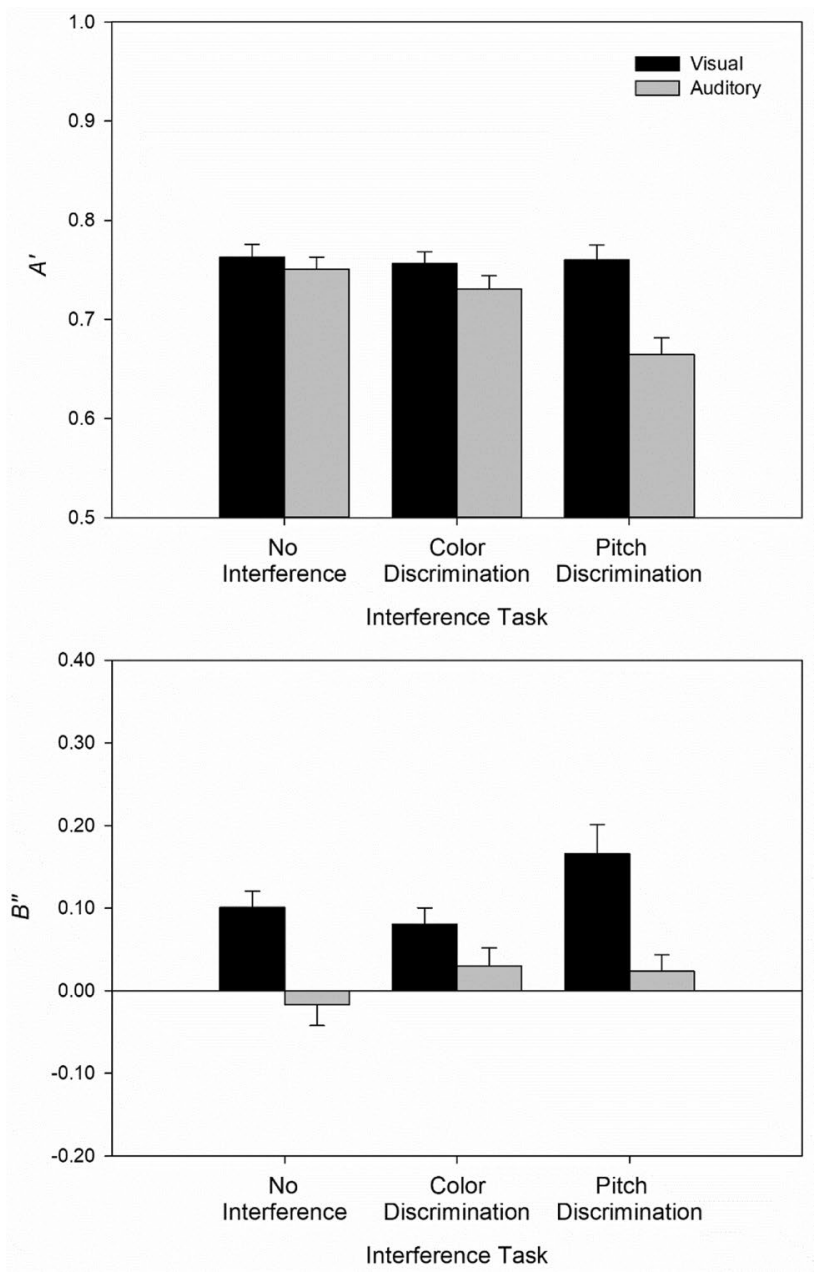

Fig. 2 Sensitivity $A^{\prime}$ (upper panel) and bias $B^{\prime \prime}$ (lower panel) as a function of interference task and duration modality. Error bars represent \pm 1 within-subject error according to Morey (2008)

duration modality was also significant, $F(1,47)=11.06$, $p=0.002, \eta_{p}^{2}=0.19$. Discrimination performance was on average slightly better for visual (0.76) than for auditory (0.72) duration stimuli. A paired sample $t$-test on discrimination performance only in the control condition revealed no difference between visual and auditory stimuli ( 0.76 vs. $0.75), t(47)=0.63, p=0.531$. Most important, the interaction between duration modality and interference task was also significant, $F(2,94)=6.43, p=0.002, \eta_{p}^{2}=0.12$. Separate ANOVAs for the two duration modalities revealed that the interference tasks impaired discrimination performance when the duration stimuli were auditory, $F(2,47)=11.02$, $p<0.001, \eta_{p}^{2}=0.12$, but not when the stimuli were visual, $F(2,47)=0.07, p=0.934, \eta_{p}^{2}<0.01$. Post-hoc Tukey contrasts indicated that pitch discrimination differed from color discrimination $(p=0.002)$ as well as no interference $(p<0.001)$, and that color discrimination did not differ from 


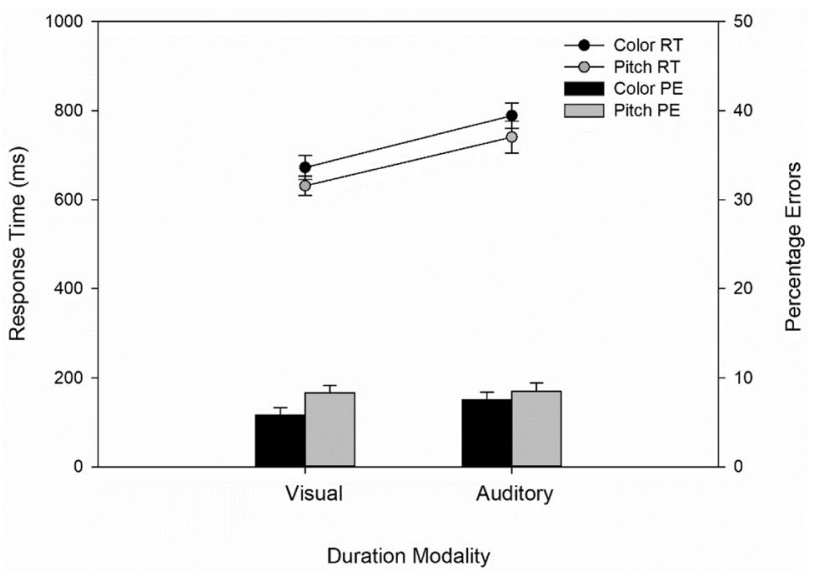

Fig. 3 Mean response time (RT, lines) and percentage of errors (PE, bars) as a function of interference task and duration modality. Error bars represent \pm 1 within-subject error according to Morey (2008)

no interference $(p=0.567)$. Together, these results show a selective interference pattern: only the auditory interference task (pitch discrimination) impaired discrimination performance and this was only the case for auditory duration stimuli.

In contrast to the previous results by Bratzke et al. (2016) and Rattat and Picard (2012), most of the $B^{\prime \prime}$ values were slightly positive (see lower panel of Fig. 2), indicating a small bias towards "different" responses $\left(B^{\prime \prime}\right.$ values can range from -1 to +1 , with negative values indicating a bias towards "same" responses and negative values indicating a bias towards "different" responses). There was a significant main effect of duration modality, $F(1,47)=20.97, p<0.001$, $\eta_{p}^{2}=0.31$, with a bias for visual $(0.12)$ and virtually no bias for auditory $(0.01)$ duration stimuli. The main effect of interference task $(p=0.091)$ and the interaction between duration modality and interference task $(p=0.092)$ were not significant.

\section{Secondary task performance (color and pitch discrimination)}

Figure 3 depicts mean RT and error rates as a function of interference task and duration modality. The analysis of mean RT revealed a main effect of duration modality, $F(1,47)=10.69, p=0.002, \eta_{p}^{2}=0.19$. Responses to the color or pitch change were faster for visual $(651 \mathrm{~ms})$ than for auditory $(765 \mathrm{~ms})$ duration stimuli. The main effect of interference task was not significant, $F(1,47)=2.21$, $p=0.144, \eta_{p}^{2}=0.01$. There was also no indication of any interaction between duration modality and interference task, $F(1,47)=0.03, p=0.854, \eta_{p}^{2}<0.01$. The analysis of error rates revealed no significant main or interaction effect, all $p s \geq 0.123$. Secondary task performance thus shows a pattern slightly different to the one of primary task performance. Auditory duration discrimination impaired response speed in the secondary task, irrespective of the modality of the interference task ${ }^{1}$.

\section{Discussion}

Previous studies regarding modality-specific interference effects in short-term retention of temporal information obtained discrepant results. Rattat and Picard (2012) observed modality-specific interference effects, that is, articulatory suppression selectively impaired retention of auditory durations and visuo-spatial tracking selectively impaired retention of visual durations. In contrast, Bratzke et al. (2016) observed a modality-independent interference effect of articulatory suppression on the retention of both, auditory and visual durations, whereas visuo-spatial tracking did not interfere with duration discrimination. The present results display a third pattern, which shares certain aspects of both previous result patterns. In line with Rattat and Picard's results, auditory interference selectively impaired the retention of auditory durations. In contrast to their results, visual interference did not impair the retention of visual durations, a result that is consistent with Bratzke et al.'s previous results. Nevertheless, the present results do not replicate the crossmodal interference effect observed by Bratzke et al., that is, discrimination of visual durations was not affected by the auditory interference task. If one assumes that the visual interference task used in the present study was simply not effective as an interference task, these results seem to match best with the previous results by Rattat and Picard and to support their conclusion that short-term memory of temporal information is modality-specific.

This interim conclusion, however, needs to be reconsidered in light of possible trade-offs between primary task (duration discrimination) and secondary task (interference tasks) performance. Analysis of secondary task performance in the present study indeed revealed crossmodal interference, which did not show up in primary task performance. That is, the retention of auditory durations resulted in slower responses (compared with visual durations) in the interference task, both for auditory (pitch) and visual (color) signals.

\footnotetext{
${ }^{1}$ To account for potentially different speed-accuracy trade-offs within secondary task performance, we additionally analyzed the balanced integration score (BIS; Liesefeld \& Janczyk, 2019), which integrates speed and accuracy. This analysis revealed essentially the same pattern as the RT analysis, with a significant main effect of duration modality, $F(1,47)=10.78, p=0.002, \eta_{p}^{2}=0.19$, and no significant main effect of interference task or interaction effect, $p \mathrm{~s} \geq 0.422$.
} 
This crossmodal interference, however, cannot explain why the auditory interference task did not impair discrimination performance for visual durations. If one assumes that participants selectively traded off secondary task performance against primary task performance in this condition, one would have expected poorer pitch discrimination performance for the visual than for the auditory discrimination task. The results, however, showed the opposite pattern. Thus, the interim conclusion in favor of modality-specific short-term memory of temporal information still holds even in light of secondary task performance.

Given the present evidence for modality-specific interference, how can we explain the discrepant results by Bratzke et al. (2016) regarding the auditory interference condition? An obvious difference between the present and previous studies is the use of an auditory interference task other than articulatory suppression. Franssen, Vandierendonck and Van Hiel (2006) investigated timing performance with empty auditory intervals under a variety of concurrent auditory interference conditions, including articulatory suppression, irrelevant speech, irrelevant tones, and music. They observed that only the "active" articulatory suppression interfered with timing performance. It is conceivable that articulatory suppression and the present auditory interference task (pitch discrimination) affect different parts of the phonological loop, namely the subvocal rehearsal process and the phonological store, respectively (see also Franssen et al., 2006). Possibly, amodal and modal representations of temporal information coexist in short-term memory (see also Bratzke \& Ulrich, 2019a; Stauffer et al., 2012) and the amodal representation can be disrupted by articulatory suppression whereas the modal representation can be disrupted by less "active" interference tasks like pitch discrimination in the present study. While these considerations are of course speculative and still cannot fully resolve the discrepancy of previous results, they highlight the importance of the present result that short-term memory of temporal information can be disrupted by an auditory interference task other than articulatory suppression.

In conclusion, previous results provided discrepant results regarding the modality specificity of interference effects between short-term retention of temporal information and modality-specific interference tasks. The present study aimed to resolve this discrepancy by analyzing possible trade-offs between primary and secondary task performance. The results are partly consistent with previous results by Rattat and Picard (2012) in favor of modality-specific short-term representations. Nevertheless, the present results suggest the possibility of coexisting amodal and modality-specific representations. Furthermore, the present study demonstrates that short-term memory of auditory durations can be disrupted by an interference task other than articulatory suppression.
Acknowledgements Open Access funding provided by Projekt DEAL. We thank Paul Kelber and Karolin Schuh for assistance with data collection. This study was supported by the Deutsche Forschungsgemeinschaft (UL 116/14-1).

\section{Compliance with ethical standards}

Conflict of interest The authors declare that they have no conflict of interest.

Ethical statements All procedures performed in studies involving human participants were in accordance with the ethical standards of the institutional and national research committees and with the 1964 Helsinki declaration and its later amendments or comparable ethical standards.

Informed consent Informed consent was obtained from all individual participants included in the study.

Open Access This article is licensed under a Creative Commons Attribution 4.0 International License, which permits use, sharing, adaptation, distribution and reproduction in any medium or format, as long as you give appropriate credit to the original author(s) and the source, provide a link to the Creative Commons licence, and indicate if changes were made. The images or other third party material in this article are included in the article's Creative Commons licence, unless indicated otherwise in a credit line to the material. If material is not included in the article's Creative Commons licence and your intended use is not permitted by statutory regulation or exceeds the permitted use, you will need to obtain permission directly from the copyright holder. To view a copy of this licence, visit http://creativecommons.org/licenses/by/4.0/.

\section{References}

Aaronson, D., \& Watts, B. (1987). Extensions of Grier's computational formulas for A' and B' to below-chance performance. Psychological Bulletin, 102, 439-442.

Baddeley, A., \& Hitch, G. (1974). Working memory. In G. Bower (Ed.), Psychology of learning and motivation (pp. 47-90). Cambridge: Academic Press.

Brainard, D. H. (1997). The psychophysics toolbox. Spatial Vision, 10, 433-436. https://doi.org/10.1163/156856897X00357.

Bratzke, D., Quinn, K. R., Ulrich, R., \& Bausenhart, K. M. (2016). Representations of temporal information in short-term memory: Are they modality-specific? Acta Psychologica, 170, 163-167.

Bratzke, D., Seifried, T., \& Ulrich, R. (2012). Perceptual learning in temporal discrimination: Asymmetric cross-modal transfer from audition to vision. Experimental Brain Research, 221, 205-210.

Bratzke, D., \& Ulrich, R. (2019a). Temporal reproduction within and across senses: Testing the supramodal property of the PacemakerCounter Model. Journal for Experimental Psychology: Human Perception and Performance, 45, 1218-1235.

Bratzke, D., \& Ulrich, R. (2019b). Temporal sequence discrimination within and across senses: do we really hear what we see? Experimental Brain Research, 237, 3089-3098.

Buonomano, D. V., \& Karmarkar, U. R. (2002). How do we tell time? The Neuroscientist, 8, 42-51.

Creelman, C. D. (1962). Human discrimination of auditory stimuli. Journal of the Acoustical Society of America, 34, 582-593. 
Franssen, V., Vandierendonck, A., \& Van Hiel, A. (2006). Duration estimation and the phonological loop: Articulatory suppression and irrelevant sounds. Psychological Research, 70, 304-316.

Gibbon, J., Church, R. M., \& Meck, W. H. (1984). Scalar timing in memory. Annals of the New York Academy of Sciences, 423, $52-77$.

Grier, J. B. (1971). Nonparametric indexes for sensitivity and bias: Computing formulas. Psychological Bulletin, 75, 424-429.

Grondin, S. (1993). Duration discrimination of empty and filled intervals marked by auditory and visual signals. Perception and Psychophysics, 54, 383-394.

Guttman, S. E., Gilroy, L. A., \& Blake, R. (2005). Hearing what the eyes see: Auditory encoding of visual temporal sequences. Psychological Science, 16, 228-235.

Ivry, R. B., \& Schlerf, J. E. (2008). Dedicated and intrinsic models of time perception. Trends in Cognitive Sciences, 12, 273-280.

Kanai, R., Lloyd, H., Bueti, D., \& Walsh, V. (2011). Modality-independent role of the primary auditory cortex in time estimation. Experimental Brain Research, 209, 465-471.

Klauer, K. C., \& Zhao, Z. (2004). Double dissociations in visual and spatial short-term memory. Journal of Experimental Psychology. General, 133, 355-381.

Kleiner, M., Brainard, D. H., \& Pelli, D. (2007). What's new in Psychtoolbox-3? Perception, 36, 1-16. (ECVP Abstract Supplement).

Liesefeld, H. R., \& Janczyk, M. (2019). Combining speed and accuracy to control for speed-accuracy trade-offs (?). Behavior Research Methods, 51, 40-60.

Morey, R. (2008). Confidence intervals from normalized data: A correction to Cousineau (2005). Tutorials in Quantitative Methods for Psychology, 4, 61-64.
Rammsayer, T., \& Ulrich, R. (2005). No evidence for qualitative differences in the processing of short and long temporal intervals. Acta Psychologica, 120, 141-171.

Rattat, A. C., \& Picard, D. (2012). Short-term memory for auditory and visual durations: Evidence for selective interference effects. Psychological Research, 76, 32-40.

Stauffer, C. C., Haldemann, J., Troche, S. J., \& Rammsayer, T. H. (2012). Auditory and visual temporal sensitivity: Evidence for a hierarchical structure of modality-specific and modality-independent levels of temporal information processing. Psychological Research, 76, 20-31.

Treisman, M. (1963). Temporal discrimination and the indifference interval. Implications for a model of the "internal clock". Psychological Monographs, 77, 1-31.

Ulrich, R., Nitschke, J., \& Rammsayer, T. (2006). Crossmodal temporal discrimination: Assessing the predictions of a general pacemakercounter model. Perception and Psychophysics, 68, 1140-1152.

Wearden, J. H., Todd, N. P. M., \& Jones, L. A. (2006). When do auditory/visual differences in duration judgements occur? Quarterly Journal of Experimental Psychology, 59, 1709-1724.

Welch, R. B., \& Warren, D. H. (1980). Immediate perceptual response to intersensory discrepancy. Psychological Bulletin, 88, 638-667.

Publisher's Note Springer Nature remains neutral with regard to jurisdictional claims in published maps and institutional affiliations. 